\title{
Kidneys control inter-organ homeostasis
}

\author{
Victor G. Puelles and Tobias B. Huber『)
}

Cockayne syndrome is a genetic disease characterized by impairment of DNA repair mechanisms, premature ageing, cachexia and kidney dysfunction. New research in a mouse model of Cockayne syndrome demonstrates that injury induces a subset of kidney proximal tubule cells to express the anorexigenic peptide GDF15. These findings link kidney injury to cachexia and highlight the role of the kidney in mediating inter-organ homeostasis.

Refers to Mulderrig, L. et al. Aldehyde-driven transcriptional stress triggers an anorexic DNA damage response. Nature 600, 158-163 (2021).

Cells of the kidney tubule have been the focus of much attention in recent years owing to the development of sodium-glucose cotransporter-2 (SGLT2) inhibitors, which target tubule epithelial cells to prevent glucose reabsorption and improve kidney outcomes in patients with type 2 diabetes ${ }^{1}$. Surprisingly, the benefits of SGLT2 inhibitors extends beyond diabetic kidney disease, with considerable benefits demonstrated on overall cardiovascular health and in patients with non-diabetic chronic kidney disease $(\mathrm{CKD})^{2}$. Although proximal tubule cells are key players in the pathophysiology of both diabetic and non-diabetic kidney diseases, the profound cardiovascular effects induced by these agents suggest that pharmacological modulation of kidney health may also have notable extra-renal and inter-organ roles. An elegant set of experiments published in Nature ${ }^{3}$ now reveals a previously unrecognized link between the kidney - through the production of growth differentiation factor 15 (GDF15) by injured proximal tubule cells - and cachexia resulting from DNA damage, highlighting the role of the kidney as a homeostatic regulator of extra-renal processes.

The study by Mulderrig and colleagues aimed to dissect potential mechanisms underlying Cockayne syndrome, an autosomal recessive neurodegenerative disorder caused by mutations in ERCC6 and ERCC8 and characterized by degenerative features including premature ageing, cachexia and kidney impairment. The protein products of ERCC6 and $E R C C 8$ contribute to a DNA damage response called transcription-coupled nucleotide excision repair. In addition to identifying endogenous formaldehyde as the cause of transcriptional stress and DNA damage, Mulderrig et al. also identified kidney proximal tubule cells as a target of formaldehyde-induced transcriptional stress. They demonstrate that damage of proximal tubule cells by endogenous formaldehyde induces a p53 response that stimulates expression of GDF15 - an anorexic hormone that contributes to progressive weight loss. Of interest, our own screen for soluble pro-cachectic factors in genetic and acquired models of CKD also detected significant increases in the expression of GDF15, along with other pro-cachectic factors such as activin $\mathrm{A}$, which was produced by a subset of fibroblasts and cells of the juxtaglomerular apparatus ${ }^{4}$. Both studies demonstrate that injured kidney cells can induce profound effects beyond their regular microenvironments - specifically, on the central nervous system (CNS) and muscle showcasing the kidney as an active participant in complex inter-organ signalling (FIG. 1).

The ability of the kidney to regulate homeostatic processes, including bone and mineral, acid-base, salt and water balance, has long been recognized. Perhaps the most notable example is its role in erythropoiesis, which is orchestrated by cortical interstitial fibroblasts in the kidney in response to hypoxia-inducible factor (HIF) - a process that relies on kidney health ${ }^{5}$. In 2021 we proposed a CKD-mediated pro-cachectic model in which the kidney releases soluble pro-cachectic factors that directly modulate muscle receptor signalling. We proposed that modulation of these signalling pathways disrupts muscle homeostasis by reducing protein synthesis and increasing protein degradation ${ }^{4}$. The work of Mulderrig and colleagues $^{3}$ now suggests that GDF15 might also indirectly facilitate cachexia by modifying appetite signals. This proposal is in line

\section{Injured kidney cells can} induce profound effects beyond their regular microenvironments ... showcasing the kidney as an active participant in complex inter-organ signalling

with a previous report showing that GDF15 triggers taste aversion in mice ${ }^{6}$ and implies that kidney-derived GDF15, perhaps together with other factors, might act as a more generalized anorexic signal, for example, in patients on dialysis. However, the precise mechanisms by which GDF15 provokes cachexia through direct effects on muscle or indirectly through CNS signalling need to be further explored. Of note, in both examples mentioned above, several further transcriptional programs were identified that might also contribute to kidney-mediated cachexia, suggesting that simultaneous, interacting and potentially redundant pathway activity is likely, which may affect the development of future therapeutic strategies that aim to target the cachectic effects of CKD and Cockayne syndrome.

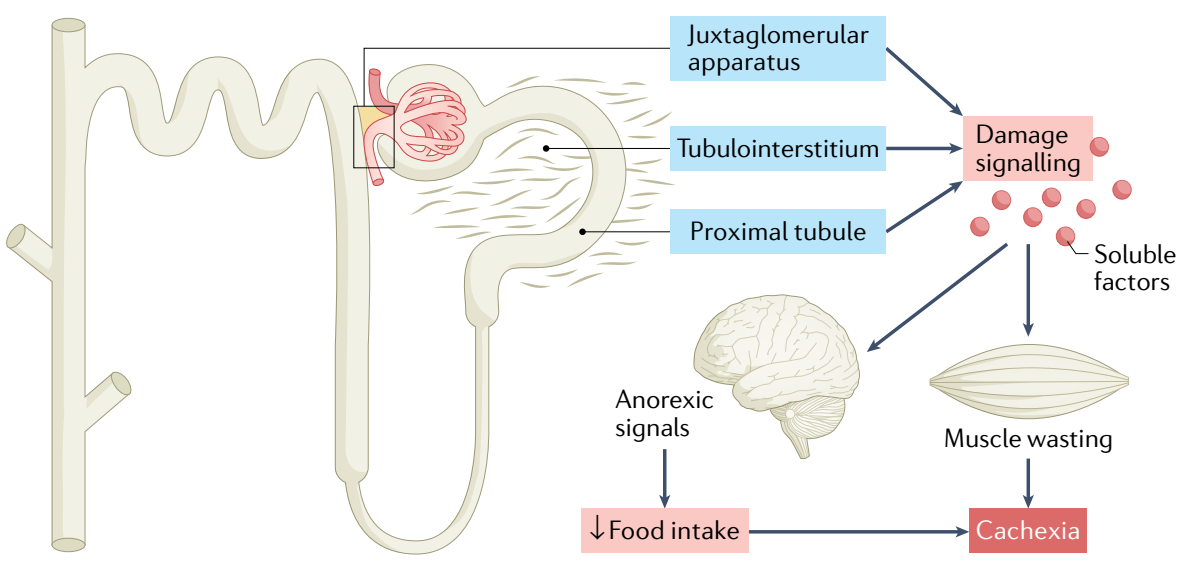

Fig. 1 | Kidneys control organ homeostasis. Kidney injury can induce the production of anorexic and muscle-wasting signals from various cell populations, resulting in cachexia. 


\section{Specific pathological changes in subpopulations of kidney cells may have notable effects outside the kidney



Proximal tubular cell injury is a central feature of acute kidney injury (AKI), including that resulting from drug-mediated toxicity ${ }^{7}$. For example, chemotherapeutic agents can induce kidney damage, with ones such as cisplatin in particular affecting the tubular epithelium ${ }^{8}$. Of note, cachexia is a well-documented complication of cancer. The findings by Mulderrig et al. and our group raise the possibility that cancer-associated cachexia might have multifactorial causes, including direct effects of the cancer itself and indirect ones from druginduced pro-anorexic factors, such as GDF15, that are induced secondary to kidney injury. Although oncological protocols may be inflexible, the implementation of nephroprotective strategies might prevent proximal tubule cell damage during chemotherapy and mitigate the pro-cachectic effects of kidney damage. AKI is also frequent among hospitalized patients, including those with COVID-19 (REF. $\left.{ }^{9}\right)$. The majority of patients seemingly recover renal function, and consideration of the potential long-term consequences of AKI is often limited. However, beyond the well-documented higher risk of CKD in patients who have recovered from an episode of AKI, these new findings also highlight the need to consider possible extra-renal long-term consequences of AKI. Current evidence clearly shows that the kidney orchestrates inter-organ communication hubs that may be unexpected and difficult to dissect, highlighting the need for further studies in this area.

GDF15 is well-known by the nephrology community; it is considered to be a promising biomarker of cardiovascular disease and diabetes-associated CKD, one that does not seem to be affected by SGLT2 inhibitors ${ }^{10}$. Thus, we can speculate that therapeutic targeting of GDF15 and other pro-cachectic factors released by the kidney might achieve multiple objectives, including amelioration of cell injury and modulation of inter-organ signalling with benefits for overall health. Furthermore, it is our hope that future studies may use the work of Mulderrig et al. ${ }^{3}$ as an example of how specific pathological changes in subpopulations of kidney cells may have notable effects outside the kidney, highlighting the central role of the kidney as a master conductor of inter-organ signalling to preserve body homeostasis.
Victor G. Puelles ${ }^{凶}$ and Tobias B. Huber (D) ${ }^{凶}$

III. Department of Medicine, University Medical Center Hamburg-Eppendorf, Hamburg, Germany.

凶e-mail:v.puelles@uke.de; t.huber@uke.de https://doi.org/10.1038/s41581-022-00549-0

1. Heerspink, H. J. L. et al. Dapagliflozin in patients with chronic kidney disease. N. Engl. J. Med. 383 , 1436-1446 (2020).

2. DeFronzo, R. A., Reeves, W. B. \& Awad, A. S Pathophysiology of diabetic kidney disease: impact of SGLT2 inhibitors. Nat. Rev. Nephrol. 17, 319-334 (2021).

3. Mulderrig, L. et al. Aldehyde-driven transcriptional stress triggers an anorexic DNA damage response. Nature 600, 158-163 (2021).

4. Solagna, F. et al. Pro-cachectic factors link experimental and human chronic kidney disease to skeletal muscle wasting programs. J. Clin. Invest. 131, e135821 (2021)

5. Schödel, J. \& Ratcliffe, P. J. Mechanisms of hypoxia signalling: new implications for nephrology. Nat. Rev. Nephrol. 15, 641-659 (2019).

6. Patel, S. et al. GDF15 provides an endocrine signal of nutritional stress in mice and humans. Cell Metab. 29 707-718.e8 (2019).

7. Scholz, H. et al. Kidney physiology and susceptibility to acute kidney injury: implications for renoprotection. Nat. Rev. Nephrol. 17, 335-349 (2021).

8. Sears, S. \& Siskind, L. Potential therapeutic targets for cisplatin-induced kidney injury: lessons from other models of AKI and fibrosis. J. Am. Soc. Nephrol. 32, 1559-1567 (2021).

9. Braun, F. et al. SARS-CoV-2 renal tropism associates with acute kidney injury. Lancet 396, 597-598 (2020).

10. Sen, T. et al. Association between circulating GDF-15 and cardio-renal outcomes and effect of canagliflozin: results from the CANVAS trial. J. Am. Heart Assoc. 10 e021661 (2021).

Competing interests

The authors declare no competing interests. 